AIAA 2002 - 1910

\title{
STRATOSPHERIC OPTICAL INTER-PLATFORM LINKS FOR HIGH ALTITUDE PLATFORMS
}

\author{
Dirk Giggenbach, Robert Purvinskis ${ }^{(*)}$, Markus Werner, Matthias Holzbock \\ German Aerospace Center (DLR), Institute of Communications and Navigation, Weßling, Germany \\ (*) ITR, University of South Australia, Mawson Lakes, Australia; currently at DLR \\ Dirk.Giggenbach@dlr.de,Robert.Purvinskis@dlr.de,Markus.Werner@dlr.de,Matthias.Holzbock@dlr.de
}

\begin{abstract}
$\underline{\text { Abstract }}$
Optical propagation simulations for the case of a laser optical link between two stratospheric High Altitude Platforms have been performed. The simulations, together with communications link budget calculations, show that high data rate communications between such platforms is feasible, at data rates up to several hundred Mbps, and optical transmit-power levels of less than $1 \mathrm{~W}$ for a $500 \mathrm{~km}$ long inter platform path. Such data rates and power levels are close to the capabilities of current technology. The simulations have included use of known parameters of the optical properties of the upper atmosphere, in particular absorption by atmospheric aerosols, and atmospheric turbulence.
\end{abstract}

\section{$\underline{\text { 1. Introduction }}$}

This paper discusses some key issues of optical interplatform links (OIPLs), where an OIPL connects two stratospheric high altitude platforms (HAPs) which serve as communications base stations for their underlying footprint area. A number of such HAP wireless communication systems based on various types of high altitude platforms - ranging from manned or unmanned aircraft through to balloons or Zeppelins or similar airships - have been proposed in the last few years [1].

Optical free space communication links are of course blocked by clouds; therefore, vertical cloud density distributions have to be taken into account.

Atmospheric attenuation is on the one hand caused by scattering effects with molecules and aerosols which can be evaluated by known formulas, while on the other hand molecular absorption spectra define certain "atmospheric windows" for the usable transmission wavelengths.
Atmospheric turbulence means local thermal disturbances which cause fluctuations of the index of refraction in the free atmosphere. This leads to diffraction-induced intensity fluctuations of the propagating wavefront, (see fig. 10), so-called speckleor scintillation-patterns

To enable the evaluation of the effects of atmospheric optical turbulence a software tool has been developed at DLR to simulate the propagation of optical waves through the open turbulent atmosphere.

In this paper we approach the concept of connecting HAPs with optical links in several sections. Firstly, we discuss the current proposals for the platforms themselves, and possible inter-platform network scenarios. This is followed by a discussion of the optical technology, emphasising the applicability of currently available techniques. The various restrictions on the optical link are then described, including geometric and atmospheric attenuation, and most importantly, atmospheric turbulence. Finally, the results of simulations are described, to give a feel for the types of services which could be available between HAPs using optical technology. These simulations have allowed estimates of communications parameters to be made from knowledge of optical properties of the upper atmosphere.

\section{Overview of Projected HAPs-Systems}

In general, the various projected HAPs systems are intended to be more or less located in particular fixed positions, except some systematic movement around their nominal position in some cases.

At the moment we identify two types of HAP-systems [2]:

Copyright () 2002 by the American Institute of Aeronautics and Astronautics Inc. All rights reserved. 
- Airships: With SkyStation being the most well-known and technically challenging representative, such airships with an operational altitude of $21 \mathrm{~km}$ would be typically unmanned, and station-keeping would be performed by electric motors and propellers.

- Airplanes: A well-known representative of a manned aircraft system concept is the $H A L O$ scheme. Here the permanent service over a given area is achieved by a $3 \times 8 \mathrm{~h}$ circling aircraft scheme operated by a pilot and a copilot on-board the aircraft powered by two fan jet engines. An unmanned solar powered airplane solution is developed by the European IST project HeliNet. Here also the airplanes are intended to operate in small circles at low speed in an altitude of $17-20 \mathrm{~km}$.

While each system has its own characteristics, all are considered useful for communications purposes, in that the operational area (communications footprint) is suitably large enough for a considerable number of users, and also the mobility of the platform itself allows advantages over current terrestrial stations.

In this paper we consider an essentially platformindependent system. We have examined the effects of platform altitude and separation on the OIPL.

\section{Scenarios for Optical Inter-Platform Links}

Various network topologies have been proposed for communication systems using HAPs, including standalone platforms, HAP-to-HAP-links via ground stations, inter-platform links, and platform to satellite links [3], [4]. The optical terminals we propose would typically be used in the last two topologies.

A case can be made for an autonomous HAP-based communication system that inherently provides full coverage to a larger region, requiring a number of "neighboring" platforms, or interconnects a number of local HAP service islands. Such a system could be efficiently realized with a network of platforms interconnected by either microwave or optical links. The feature of incorporating an optical trunk subnetwork is mentioned in [5], and optical networking concepts for this environment are discussed in [4].

The possible scenarios for IPLs include the following:

- A densely meshed metropolitan HAP network

- Connections between remote HAPs (over sea / other remote locations)
- non-permanent service provision via HAPs (e.g. for major sporting events, natural disasters)

- HAP-to-GEO or -LEO sat. communications

Compared with microwave links, the optical technology provides a number of advantages: smaller beam divergence increases reception power significantly; optical frequencies do not cause interference with other communication devices from other systems or on board the HAP; the optical link is fairly tap-proof; the optical terminal can be much smaller and less power consuming than its microwave counterpart; in principle, there is virtually no limitation for the data rates that can be carried. Disadvantages are the susceptibility to clouds and optical turbulence (as described below) and the attenuation of certain wavelength regions by the air molecules.

We have investigated scenarios with different HAP altitudes and distances to show the consequences of different atmospheric and geometric parameters. Two scenarios are depicted in fig. 1 . The 'graze height' is the minimum altitude of the direct path between two HAPs. The minimum possible graze height is determined by the cloud ceiling as discussed in section 5.1.

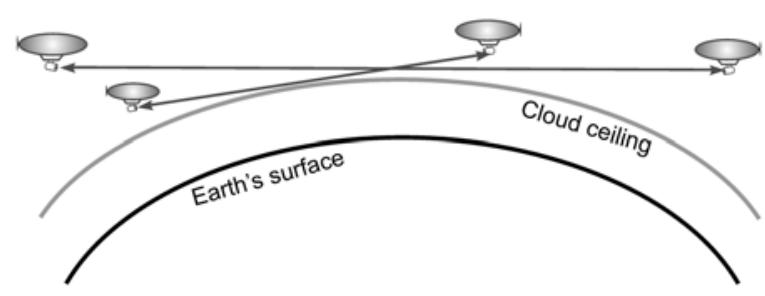

Fig. 1: Scenarios for different HAP altitudes an distances of stratospheric links above the cloud ceiling

\section{Technology of Optical Free-Space Communications Links (OFCL)}

\section{$\underline{4.1 \text { Transmission Technology }}$}

Direct Detection with on/off-keying, as known from terrestrial fiber-optical transmission, has the advantage of a range of components with proven reliability e.g. laser diodes, fiber amplifiers, detectors and receiver electronics. Even if, according to theory, a mean 10 incoming photons per bit are sufficient for an uncoded Bit-Error-Rate (BER) of $10^{-9}$, in practical systems using standard APD-detectors (avalanche photodiode), the receiver sensitivity is usually no better than 100 Photons 
per bit. This is due to thermal receiver noise and other degrading electronic effects. Another disadvantage is the susceptibility to background radiation from celestial bodies or clouds that are located in line-of-sight behind the transmitter, which causes additional shot-noise on the detector. This can be reduced by lossy optical filters which have a typical minimum broadness of $3 \mathrm{~nm}$. Despite these disadvantages, direct detection provides a quite robust technique that has already been demonstrated in space [6].

Another option for transmission is coherent modulation - e.g. homodyne binary phase-shift-keying (BPSK) - as has been demonstrated with 18 Photons per bit at $\mathrm{BER}=10^{-9}$ [7]. The effect of background radiation can be neglected due to the extremely small noise bandwidth of the homodyne receiver which is in the order of the data-bandwidth (e.g. $1 \mathrm{GHz}$ signal bandwidth corresponds to only about $0.0035 \mathrm{~nm}$ optical bandwidth for $1 \mu \mathrm{m}$ wavelength). However, coherent transmission is very susceptible to the phase noise and wavefront distortion caused by atmospheric turbulence [8].

Pulse-Position-Modulation (PPM) has been promoted as an extremely efficient incoherent transmission scheme [9]. However, constraints in laser technology and susceptibility to background radiation prevent its implementation for multi-megabit systems.

Therefore, here we will consider direct detection systems based on experimental work as described in [10]. There a system sensitivity of an average 200 photons per bit (i.e. 400 photons per on-bit) has turned out to be a realistic value. Further improvements and experiments may lead to robust coherent free-space transmission systems which then will provide more than $10 \mathrm{~dB}$ sensitivity enhancement.

\section{$\underline{4.2 \text { Possible Wavelengths and Transmitter Power }}$}

Typical wavelengths for free-space communications include laserdiodes around $800 \mathrm{~nm}, \mathrm{Nd}-\mathrm{YAG}$-lasers at $1064 \mathrm{~nm}$, and laserdiode-technology adopted from fibercommunications at $1300 \mathrm{~nm}$ and $1550 \mathrm{~nm}$.

The wavelength for an atmospheric free space optical communications system must be chosen using the following criteria:

- commonly available, compact and efficient light sources with sufficient power and modulation capability
- detectors with sufficient sensitivity and low noise levels

- inexpensive, readily available and reliable optical materials and components

- $\quad$ eye-safe light sources, if applicable

- wavelengths not impaired by atmospheric absorption lines

- low external natural emissions near the wavelength (from Sun, astronomical sources, or the atmosphere itself) to reduce natural background interference

From these criteria it follows that the wavelength should be in the infrared region, where the common wavelength for fiber communications of $1550 \mathrm{~nm}$ provides both a range of available inexpensive but reliable components and a better level of eye-safety compared to the near-IR region (eye-safety might be an issue with manned HAPs). Additionally, the effect of Rayleigh scattering strongly diminishes with longer wavelengths and the emission from natural backgroundlight sources (which impairs Rx-performance) is very low. The higher diffraction-limited divergence of longer wavelengths is not disadvantageous here as a higherthan-diffraction-limited divergence will be necessary due to PAT-limitations.

Maximum transmitter power depends on bandwidth and the modulation technology. Direct modulation of high power laser diodes up to several hundred MBps, providing a mean transmit power of $500 \mathrm{~mW}$, has already been demonstrated [11]. A more sophisticated and efficient way is to use optical amplifiers for the modulated signal. This would allow several Watts of Tx-power with data-rates up to the GBps-range.

\section{$\underline{4.3 \text { Transmission and Reception Terminals - Beam }}$}

Divergence and Field-of-View

Current technology exists for linking satellites optically (Optical Inter Satellite Links, OISL), which should be suitable for atmospheric OIPLs.

We consider a large divergence of the transmitter beam useful to cope with the platform vibrations and attitudecontrol limitations of a typical HAP. For these largerthan-diffraction-limited divergence angles, a large transmitter aperture is not required. This would allow quite small Tx-terminals. When using the same telescope for reception, however, the aperture area defines collected power and thus should be as large as possible. Therefore we define a technically feasible telescope size of $20 \mathrm{~cm}$ diameter. This aperture can consist of one standard achromatic lens. 
By using data from OISL terminals for the beamtracking ability (which is in the range of several $\mu \mathrm{rad}$ ) and making estimates for the attitude-control of typical HAPs, we can assume the divergence-angle for the Tx and the field-of-view (FOV) for the Rx of $300 \mu \mathrm{rad}$ as technically feasible. Closed-loop control of both terminals allows a PAT control bandwidth of more than $50 \mathrm{~Hz}$ due to the short round-trip transmission time.

\section{Atmospheric Effects on OIPLs and other $\underline{\text { Restrictions }}$}

The largest difference between free-space optical and microwave transmission technology occurs in the types of interaction between an optical beam and the atmosphere. It is therefore important to fully understand the effects of atmospheric structure, attenuation, and turbulence on the propagation of an optical beam. In addition, geometrical concerns and background light levels will also affect the design of an optical HAPHAP link.

\section{$\underline{5.1 \text { Structure of Earth's Atmosphere }}$}

The earth's atmosphere consists of several distinct layers with the troposphere at the bottom. Usually all weather phenomena (and thus cloud coverage) happens inside the troposphere which has a negative temperature coefficient of average $-1 \% / 100 \mathrm{~m}$ over altitude. The tropopause is defined by the reversing of the temperature coefficient which then is positive inside the stratosphere. The lower bound of the stratosphere varies from below $8 \mathrm{~km}$ at the poles, up to $18 \mathrm{~km}$ at the equator.

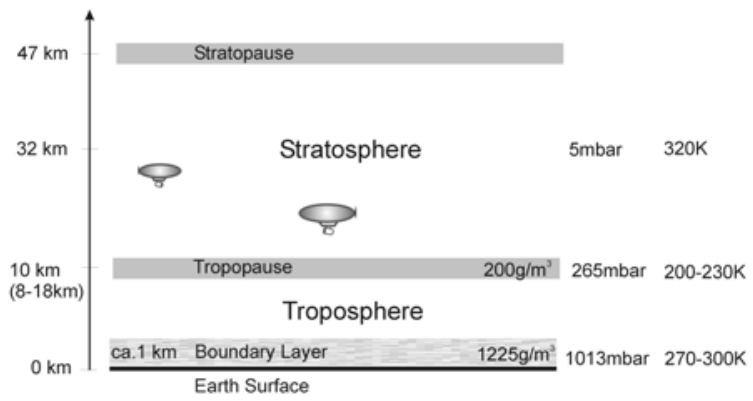

Fig. 2: Structure of Earth's atmosphere

Several years of satellite data has been gathered concerning the distribution and probability of clouds on a global scale (see fig. 4). This has included satellite measurements of cloud heights and opacities. Interestingly, although cloud thickness is not easily directly measurable, maximum cloud height (cloud ceiling) data is available - the very parameter required to design HAP-HAP links.

One study [12] has presented measured average maximum cirrus heights (for temperate latitudes) up to $14 \mathrm{~km}$. Furthermore, extensive satellite cloud measurements have sampled thin cirrus clouds at altitudes above $16 \mathrm{~km}$ [13]. The minimum graze height for any HAP-HAP link would have to take into account local climatic conditions (i.e. tropopause height), however we consider a minimum graze height of $13 \mathrm{~km}$ as a valid limit for mid-latitude HAPs-links.

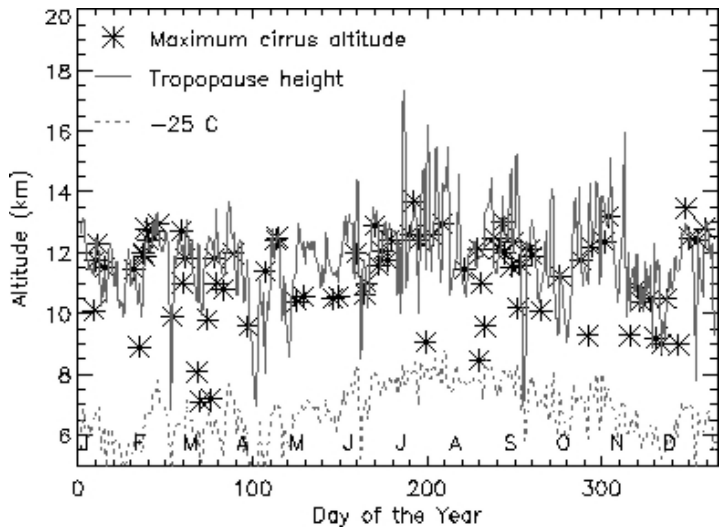

Fig. 3: Cloud ceiling (maximum cirrus altitude) measured above south of France (after [12])

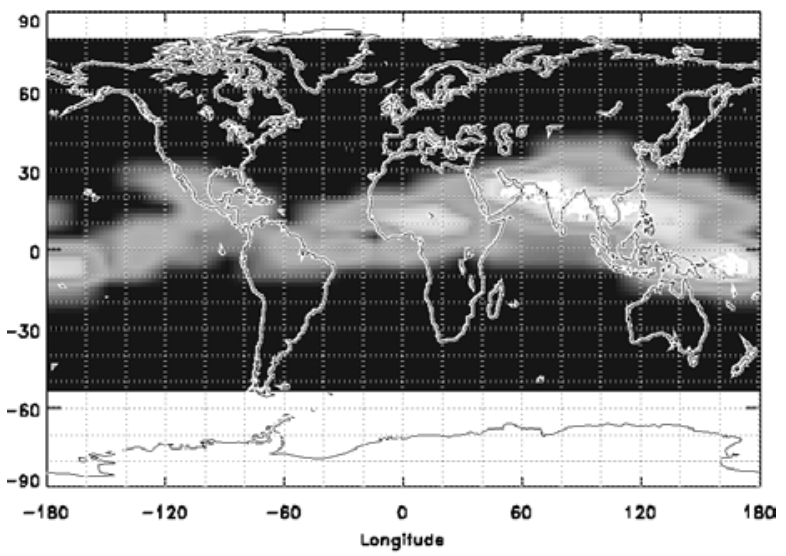

Fig. 4: Probability of cloud-distribution at $16 \mathrm{~km}$ altitude in July; cloud density represented by brightness (after [13], SAGE-satellite data)

\subsection{Geometric Restrictions and Link Distances}

In a purely parametric sense, there is no reason why two HAPs cannot be at different heights. This could be used during the establishment of platform station-keeping, 
avoidance of weather at lower HAP altitudes, and also to change the size of the footprint or power budget. The exact operational reasons depend on the particular HAP-HAP scenario used in the design. In practice, however, the view from the higher HAP may have higher background interference, as it will see the lower HAP with clouds or the Earth in the background. We therefore consider only HAPs at equal heights in the rest of our discussion.

For a given graze height of $13 \mathrm{~km}$, it can be seen in fig. 5 that the link distance will vary between approximately 300 and $1000 \mathrm{~km}$, depending on the heights of the two platforms.

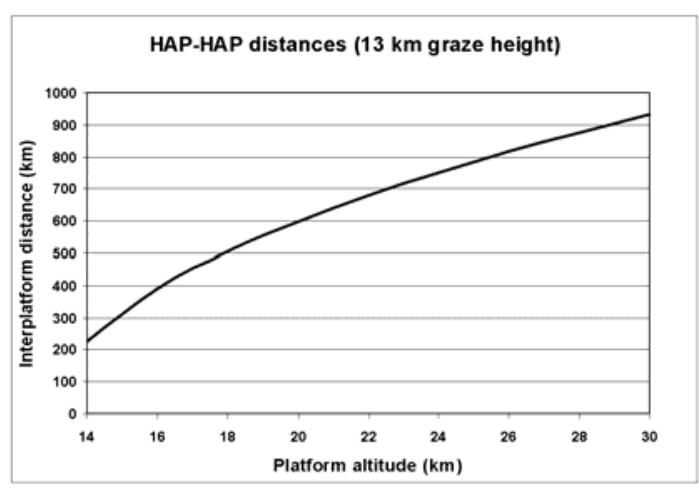

Fig. 5: Maximum possible distance for two HAPs at same altitude for an OIPL above the $13 \mathrm{~km}$ cloud ceiling

\subsection{Stratospheric Attenuation}

Attenuation in the stratosphere is caused by mainly three effects: molecular absorption, scattering by droplets or ice crystals and in clouds (in extreme weather situations, like thunderstorms, clouds can break through the tropopause). A rare phenomenon which is connected to very low temperature inside the stratosphere are polar stratospheric clouds (PSC) which have only low optical thickness and need not be taken into account for medium or tropical latitudes.

The transmitted power is calculated using the altitudedependent attenuation coefficient $\alpha(h)$ for the Beer's law according to

$$
P_{\text {out }}(L, h)=P_{\text {in }} \cdot e^{-\alpha(h) \cdot L}
$$

Fig. 6 shows an estimate for the stratospheric attenuation coefficient over a broader optical spectrum which also clarifies the advantage of $1550 \mathrm{~nm}$ over other typical communications laser wavelengths.

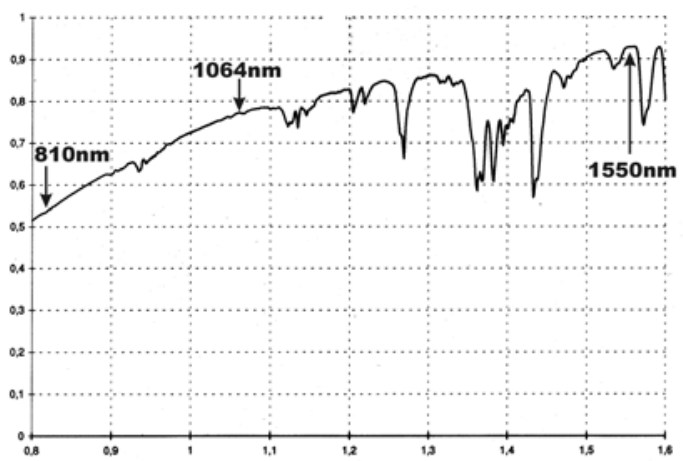

Fig. 6: Simulated atmospheric transmittance (in arbitrary units) at $15 \mathrm{~km}$ altitude in midlatitude summer from $0.8 \mu \mathrm{m}$ to $1.6 \mu \mathrm{m}$ without aerosols

Around a certain laser wavelength the molecular absorption spectrum shows very distinct lines as shown in fig. 7. The knowledge of the exact location of these absorption lines, which are due to $\mathrm{CO}_{2}$ and $\mathrm{H}_{2} \mathrm{O}$, allows appropriate selection of the laser wavelength to reduce atmospheric absorption.

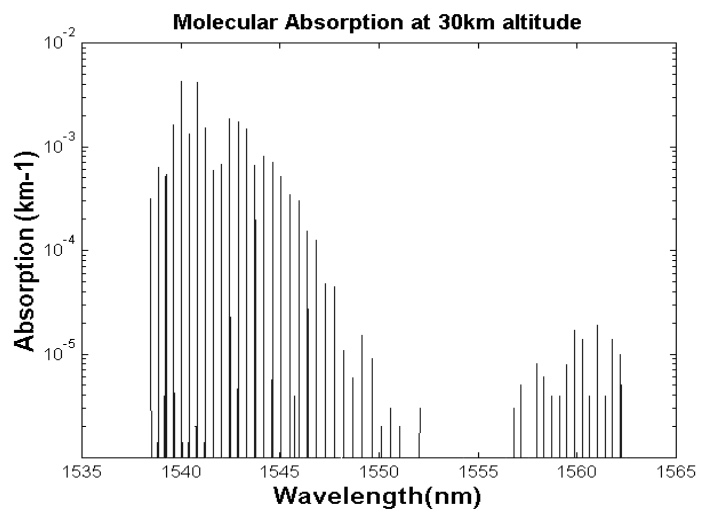

Fig. 7: Molecular absorption lines of stratospheric gases around $1555 \mathrm{~nm}$ at an altitude of $30 \mathrm{~km}$

Note that a spectral 'window' is available at $1552.4 \mathrm{~nm}$ once the altitude is raised above $10 \mathrm{~km}$ (fig. 8). This is because of the changing composition and reduction of pressure broadening with altitude. 


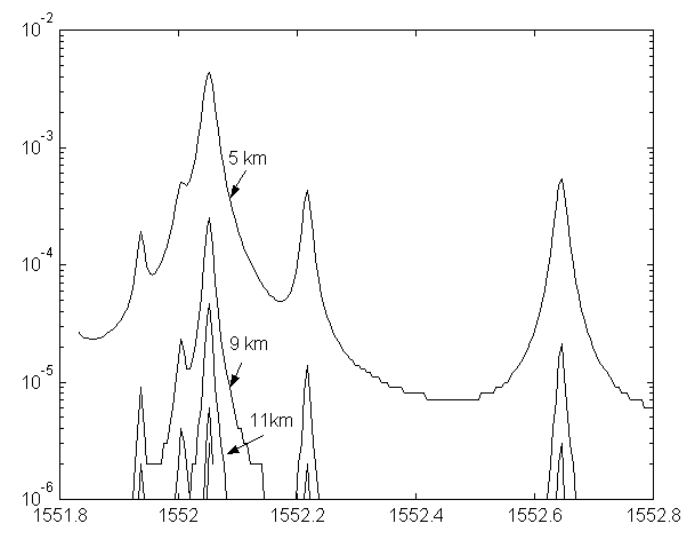

Fig. 8: Variation of molecular absorption coefficient with altitude around $1552 \mathrm{~nm}$

A more dominating effect on optical beams in the upper atmosphere is produced by the scattering of beams by stratospheric aerosols. The concentrations of such aerosols vary, depending mainly on worldwide volcanic activity, and can change the absorption coefficient of the atmosphere by several orders of magnitude. Fig. 9 shows the absorption at $1550 \mathrm{~nm}$ due to aerosols in the stratosphere, plotted against altitude, for several states of volcanic activity. This is based on well-known atmospheric models [14], [15], and [16].

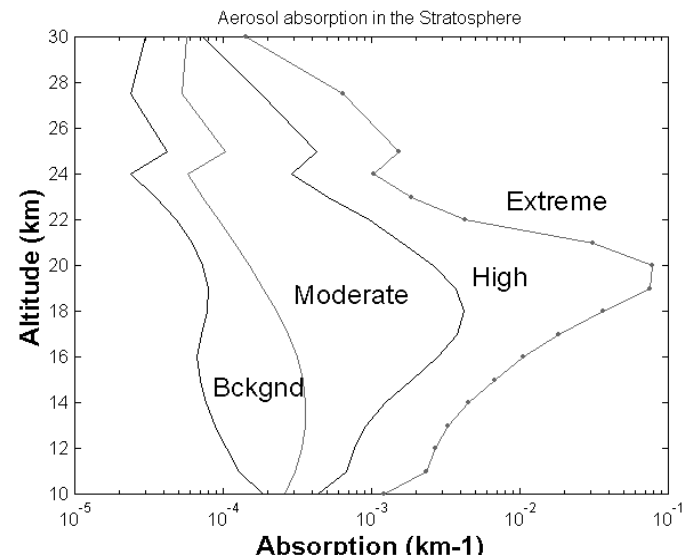

Fig. 9: Total absorption coefficient vs. altitude at $1550 \mathrm{~nm}$ for varying levels of volcanic activity

\subsection{Effects of Air-Turbulence on Stratospheric OIPLs}

The major difference between current optical satellite up- and downlinks and HAP-HAP links is the long horizontal path length. Current systems and studies of optical atmospheric turbulence (including most analyses used for adaptive optics in astronomy) consider only relatively short path lengths through the atmosphere, albeit through the highly turbulent lower troposphere [17]. In the case of HAP-HAP crosslinks, the beam propagates through a thinner, less dense medium, but is subject to long path lengths (up to $1000 \mathrm{~km}$, compared to the $20 \mathrm{~km}$ or so total turbulent path length of a vertical uplink). The result of this long turbulent path is a random intensity distribution at the receiver as demonstrated in fig. 10. Of course the Rx-phase is also randomly distorted, which is not of interest for a direct detection receiver.

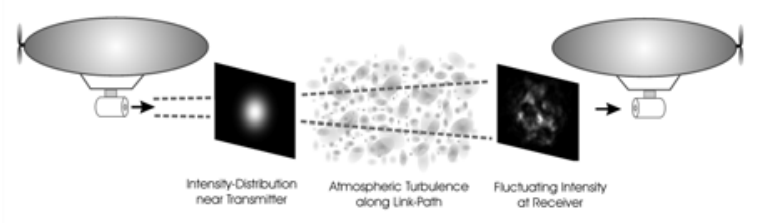

Fig. 10: Speckle patterns in the received optical field originating from index-of-refraction turbulence along the beam-path (telescope not to scale)

It is generally recognised that optical scintillation decreases with increasing wavelength due to the lesser impact of optical path differences on the phase of the optical field, another argument in favour of $1550 \mathrm{~nm}$ compared to shorter wavelengths.

The structure of the atmospheric turbulence in the stratosphere has several effects on a propagating laser beam. Firstly, turbulence nearest the transmitter will divert the beam away from the receiver, which has a great affect on the pointing accuracy due to the long propagation distance. Secondly, as the beam propagates, it is broken up by destructive interference into smaller speckles, which then are further affected by the atmosphere. The scale size of such speckles are important in the design of the receiver antenna, since it is preferable to have more than one speckle (for direct detection modulation) on the receiver at any one time. These aspects are treated in more detail in section 7. Finally, it must be remembered that the atmosphere, even at these altitudes, is not static, and high altitude winds will move the turbulence structures along and across the beam over relatively long time periods compared to the bitrate. This causes received power fades of the order of several milliseconds.

In addition to the atmosphere itself, the motion of the platform also impacts upon the temporal spectrum of power fluctuations. 


\section{OIPL-Scenarios and Parametric Link Budget}

We have identified five typical OIPL-scenarios with different HAPs-altitudes and -distances. Graze height is the minimum height of the optical link above the earth surface.

\begin{tabular}{|l|c|c|c|}
\hline \multicolumn{1}{|c|}{ scenario } & $\begin{array}{c}\text { HAPs- } \\
\text { altitude }\end{array}$ & $\begin{array}{c}\text { OIPL- } \\
\text { distance }\end{array}$ & $\begin{array}{c}\text { graze- } \\
\text { height }\end{array}$ \\
\hline high-long (HL) & 30 & 932 & 13 \\
\hline high-short (HS) & 30 & 461 & 25.7 \\
\hline medium-short (MS) & 24 & 375 & 21.25 \\
\hline low-long (LL) & 18 & 506 & 13 \\
\hline low-short (LS) & 18 & 253 & 16.74 \\
\hline
\end{tabular}

Table 1: Investigated scenarios (all values in $\mathrm{km}$ )

For the high and the low platform-altitudes we chose the maximum possible OIPL-distance (as limited by the cloud-ceiling) and as a second scenario half of this maximum distance.

The link distances listed in table 1 do not take into account beam deviation caused by atmospheric refraction, which has a small effect of raising the apparent target HAP position slightly higher above the visible horizon. The amount of refraction is dependent on the graze height, and in particular, the density and temperature of the atmosphere at the graze point.

The diameter of the usable area for communication services underneath each HAP (footprint) depends on a required minimum ground terminal elevation. These are stated in table 2 for various scenarios.

\begin{tabular}{|c|c|c|c|}
\hline \multirow{2}{*}{ scenario } & \multicolumn{3}{|c|}{ minimum ground-antenna elevation } \\
\cline { 2 - 4 } & $\mathbf{2 0}^{\circ}$ & $\mathbf{1 0}^{\circ}$ & $\mathbf{5}^{\circ}$ \\
\hline HL \& HS & 161 & 316 & 548 \\
\hline MS & 129 & 256 & 454 \\
\hline LL \& LS & 98 & 195 & 346 \\
\hline
\end{tabular}

Table 2: Footprint diameters (in $\mathrm{km}$ ) for the different HAPs-altitudes depending on minimum ground-antenna elevation angle

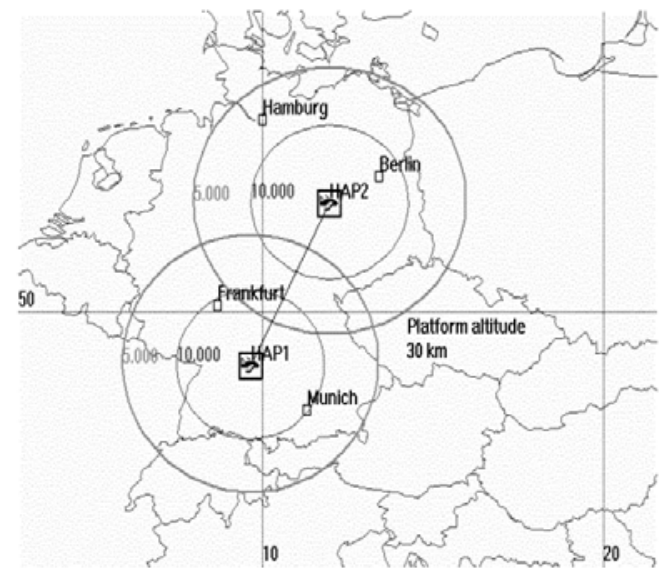

Fig. 11: Example of footprint areas $\left(5^{\circ}\right.$ and $10^{\circ}$ ground elevation) of two HAPs with $400 \mathrm{~km}$ distance

The actual size of the footprint used in developing a complete HAP system would require a more detailed assessment of the required uplink elevation angle, which is outside the scope of this paper [2].

Fixed parameters for the link budget are the $300 \mu \mathrm{rad}$ full divergence angle from the transmitter, the wavelength of $1550 \mathrm{~nm}$, and $20 \mathrm{~cm}$ Rx-aperture diameter. Table 3 shows some of the scenariodependent parameters for the link budget calculation.

\begin{tabular}{|c|c|c|}
\hline scenario & $\begin{array}{c}\text { free-space loss } \\
\text { in dB }\end{array}$ & $\begin{array}{c}\text { atmospheric } \\
\text { attenuation in } \mathbf{~ d B}\end{array}$ \\
\hline HL & -63 & -6.6 \\
\hline HS & -57 & -0.6 \\
\hline MS & -55 & -1.6 \\
\hline LL & -58 & -4.1 \\
\hline LS & -52 & -4.1 \\
\hline
\end{tabular}

Table 3: Link-budget parameters

The atmospheric attenuation values are for high volcanic activity and thus represent a nearly worst case. Additional to those mentioned in table 3 other link budget parameters include: data-rate, transmit-power, telescope-transmission losses (-3dB), PAT-losses $(-3 \mathrm{~dB}), \mathrm{Rx}$-detector-efficiency $(-2 \mathrm{~dB})$, a receiver quality of an average 200 photo-electrons per Bit, and other implementation losses $(-3 \mathrm{~dB})$. These are realistic values based on experimental results from other research [11]. Scintillation losses will be treated in more detail in section 7. Turbulent beam-spreading losses can be neglected due to the high divergence of the transmitter beam, which is much larger than the diffraction limit. 
The impact of background light is negligible in almost all cases of celestial bodies except for the sun which would usually impair transmission while it is crossing the line-of-sight of the receiving telescope. The sun's disc fills only $5 \times 10^{-6}$ of the sky, which affects link availability occasionally. Furthermore, these shutdown periods can be exactly predicted. However, optical shielding is needed for near-sun angles of view to avoid reflections of stray sunlight inside the Rx-telescope.

\section{Simulation of Optical Atmospheric Propagation and OIPL-Performance}

We simulated the atmospheric propagation of all five scenarios to calculate their communication link budgets. The analytical theory for calculation of statistical parameters of atmospheric optical propagation is treated in detail in [17] and [18]. Here we present the results of a numerical beam propagation simulation based on the phase-screen method as described in [19], where turbulent path volumes are regarded step-wise as phasedistorting planes with free-space diffractive propagation between them. Simulations are performed using the MatLab-Toolbox "PILab" which was developed at DLR [20].

The altitude profile for the parameter $\mathrm{C}_{\mathrm{n}}{ }^{2}$ (which scales the atmosphere's index-of-refraction structure function) is generated using the Hufnagel-Valley model [17]. This analytical function is partly supported by measurements of stratospheric turbulence (fig. 12). This altitudeprofile is mapped to the path-profile of each scenario to provide the basis for the propagation simulation.

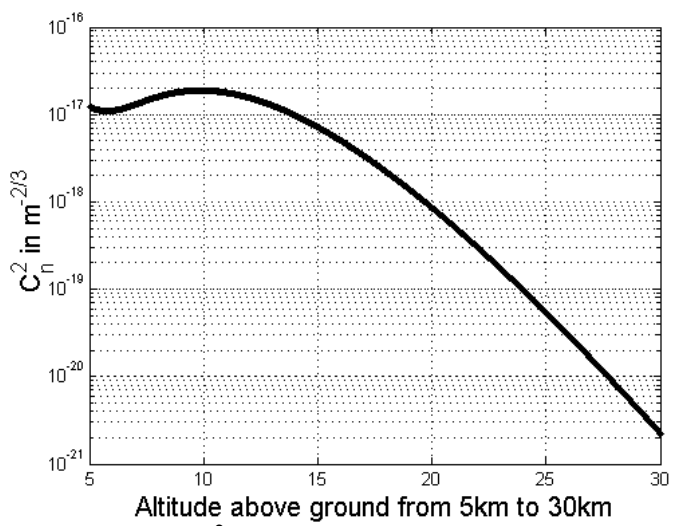

Fig. 12: Used $\mathrm{C}_{\mathrm{n}}{ }^{2}$-profile over altitude
The simulation produces samples of the Rx-field which then are converted into the intensity distributions as shown in fig. 13.

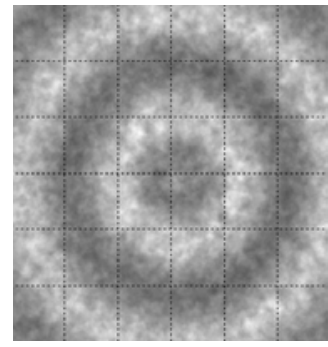

HS-scenario

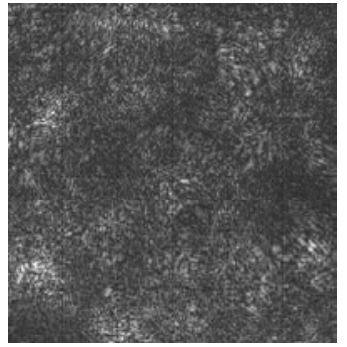

HL-scenario
Fig. 13: Typical simulated Rx-intensity distributions (shown area is $24 \mathrm{~m} \times 24 \mathrm{~m}$ )

For the scenario with the fewest turbulence-impact (HS), the far-field diffraction pattern from the transmitting aperture can still be seen, while in those cases that go down to the lowest graze height, the scintillations dominate over the diffraction pattern. The "Long-distance"-scenarios are impacted much more strongly by turbulence than the "Short"-ones, even though the HS distance is longer than the LL. This is because the stronger turbulence near the graze-height limit at $13 \mathrm{~km}$ dominates over long path lengths.

To convert these intensity distributions into $\mathrm{Rx}$-power over time, an integrating aperture area of $20 \mathrm{~cm}$ diameter is scanned over the matrix with a given lateral wind speed of $20 \mathrm{~m} / \mathrm{s}$ which is a typical value for stratospheric altitudes. Figs. 14 to 16 show statistic evaluations of the $\mathrm{Rx}$-power for the HL-scenario.

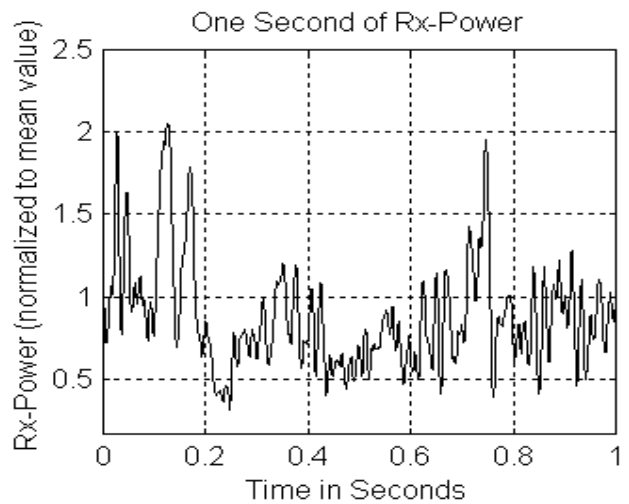

Fig. 14: Typical Rx-power vs. time for a lateral windspeed of $20 \mathrm{~m} / \mathrm{s}$ 
The temporal spectrum of Rx-power fluctuations is usually negligible beyond $100 \mathrm{~Hz}$ (see fig. 15).

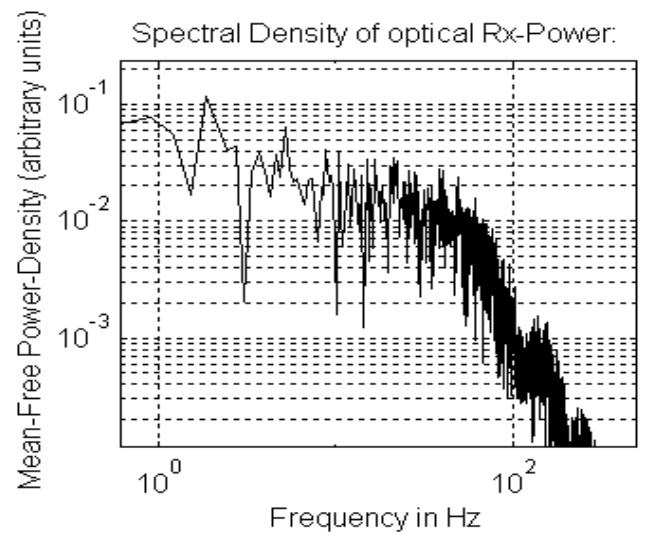

Fig. 15: Typical spectrum of received power fluctuations in $\mathrm{Hz}$ for $20 \mathrm{~m} / \mathrm{s}$ lateral wind speed

Remarkable in fig. 16 is the benefit from the apertureaveraging effect, because the Rx-aperture is larger than the smallest structures in the speckle pattern. The PDF would have values further down to zero with decreasing aperture size.

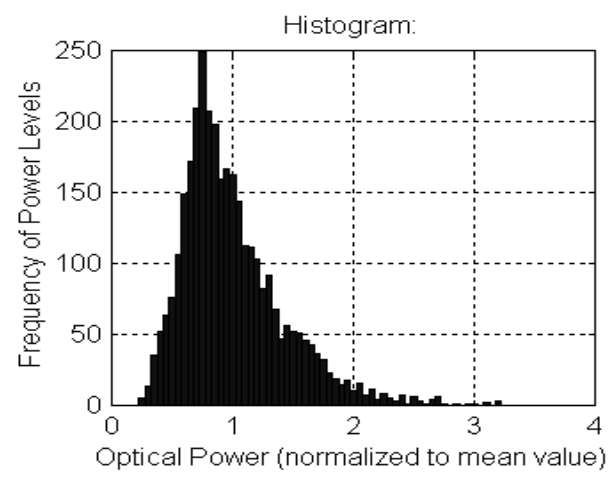

Fig. 16: Typical probability density function of received power

As a parameter for the scintillation losses we take the power penalty 'scintillation loss' for a fading probability of less than $1 \%$ as stated in table 4 , i.e. when spending the calculated power margin for scintillation losses, then during $99 \%$ of the time the received power level is above the required link budget threshold. The remaining $1 \%$ time below the fading level is expected to be covered by forward error correction coding (FEC) in combination with a sophisticated interleaving scheme to spread the long fading intervals evenly over the bit stream.

\section{Results}

Taking into account the simulated power penalties for scintillation compensation and the link budget parameters from section 6 we can scale the required Txpower for zero link margin according to table 4 for three typical networking data rates.

\begin{tabular}{|c|c|c|c|c|}
\hline \multirow{2}{*}{ scenario } & scint. loss & \multicolumn{3}{|c|}{ required mean Tx-power for... } \\
\cline { 3 - 5 } & in dB & 10MBps & 100MBps & 622MBps \\
\hline HL & -5 & $100 \mathrm{~mW}$ & $1 \mathrm{~W}$ & $6.5 \mathrm{~W}$ \\
\hline HS & -1.7 & $2.6 \mathrm{~mW}$ & $26 \mathrm{~mW}$ & $175 \mathrm{~mW}$ \\
\hline MS & -3.7 & $3.5 \mathrm{~mW}$ & $35 \mathrm{~mW}$ & $218 \mathrm{~mW}$ \\
\hline LL & -5.5 & $17 \mathrm{~mW}$ & $170 \mathrm{~mW}$ & $1 \mathrm{~W}$ \\
\hline LS & -5.8 & $4.7 \mathrm{~mW}$ & $47 \mathrm{~mW}$ & $290 \mathrm{~mW}$ \\
\hline
\end{tabular}

Table 4: Link Budget - Required Tx-power at different data-rates for zero link margin with $1 \%$ fading probability

These required Tx-power values are well within the technically feasible range. This shows, that OIPLs are a very useful option when designing HAP-based communications systems.

\section{Outlook}

We have shown the feasibility of connecting stratospheric high altitude platforms with optical freespace technology to provide a high data-rate backbone for future communications systems.

More information is needed about the upper atmosphere's turbulence and aerosol attenuation. Additionally, wind profiles would have to be treated in detail as they severely affect the temporal fade behaviour and thus define the requirements of error correction coding. Realistic vibration spectra and attitude stability of HAPs need to be evaluated after operational tests of actual platforms. This will further define the parameters of a free-space optical link between two platforms.

The applicability of coherent transmission with special techniques to cope with distorted wavefronts has to be evaluated. Coherent technology is preferable in the long term because of its increase of sensitivity and the negligible susceptibility to background light.

Further scenarios to be addressed include optical HAPto-Satellite links. This would allow the direct connection to high data rate satcom systems and inter continental (world wide) data links. 


\section{Acknowledgements}

We want to thank our colleagues from the Institute of Atmospheric Physics, DLR, for their help with the atmospheric attenuation coefficients (especially Dr. B. Mayer). R. Purvinskis thanks the University of South Australia for support during his time at DLR.

\section{List of Abbreviations}

$\begin{array}{ll}\text { APD } & \text { Avalanche Photo Diode } \\ \text { BPSK } & \text { Binary Phase-Shift Keying } \\ \text { FOV } & \text { Field-of-View } \\ \text { FEC } & \text { Forward Error Correction } \\ \text { HAP } & \text { High Altitude Platform } \\ \text { OIPL } & \text { Optical Inter-Platform Link } \\ \text { OISL } & \text { Optical Inter-Satellite Link } \\ \text { PAT } & \text { Pointing, Acquisition, and Tracking } \\ \text { PPM } & \text { Pulse-Position Modulation } \\ \text { Rx } & \text { Receiver } \\ \text { Tx } & \text { Transmitter }\end{array}$

\section{References}

J.N. Pelton, "Overview of high altitude platform systems (HAPS) and their future relation to satellite networks", in Proceedings $18^{\text {th }}$ AIAA International Communications Satellite Systems Conference (ICSSC'00), Oakland, CA, USA, pp. 259-266, Apr. 2000.

D. Grace, et al, "Providing multimedia communications services from high altitude platforms", Int. J. Sat. Com. Vol.19 p. 559, 2001

U. Drcic, G. Kandus, M. Mohorcic, T. Javornik, "Interplatform link requirements in the network of high altitude platforms", in Proceedings $19^{\text {th }}$ AIAA International Communications Satellite Systems Conference (ICSSC'01), Toulouse, France, paper No. 288 (CD-ROM), Apr. 2001.

N. Karafolas, "The case of optical networking using stratospheric platforms", in Proceedings $19^{\text {th }}$ AIAA International Communications Satellite Systems Conference (ICSSC'01), Toulouse, France, paper No. 39 (CD-ROM), Apr. 2001.
Y. Hase, G. Wu, R. Miura, "Wireless communication systems using stratospheric platforms", in Proceedings $18^{\text {th }}$ AIAA International Communications Satellite Systems Conference (ICSSC'00), Oakland, CA, USA, pp. 251-258, Apr. 2000.

[6] M. Reyes, Z. Sodnik, P. Lopez, A. Alonso, T. Viera, G. Oppenhauser, "Preliminary results of the in-orbit test of ARTEMIS with the Optical Ground Station", Proc. of SPIE 2002, Vol. 4635

B. Wandernoth, "5 Photon/bit low complexity 2MBit/s PSK transmission breadboard experiment with homodyne receiver applying synchronization bits and convolutional coding", ECOC '94, 20th European Conference on Optical Communications, Firenze, Sep. 1994

D. Giggenbach,"Wavefront Measurements at ESA's Optical Ground Station and Simulation of Heterodyne Receiver Performance", Proceedings of SPIE 2000, Vol. 3932

[9] J. Hamkins, J. Ceniceros, "The Capacity of Avalanche Photodiode-Detection Pulse Position Modulation", Proc. of SPIE Vol. 3932 (2000)

[10] D. Giggenbach, F. David, et al, "Measurements at a $61 \mathrm{~km}$ near-ground optical transmission channel", Proc. of SPIE 2002, Vol. 4635

[11] F. David, D. Giggenbach, et al, "Preliminary Results of a $61 \mathrm{~km}$ Groundto-Ground Optical IM/DD Data Transmission Experiment", Proc. of SPIE 2002, Vol. 4635

[12] Goldfarb, L. et al, "A climatological study of cirrus clouds by lidar at OHP" Proc. SPARC $20002^{\text {nd }}$ Assembly of the SPARC/WRCP Project, Argentina, Nov. 2000

[13] L. Thomason, "SAGE II Data products website", http://www-sage2.larc.nasa.gov/ data/cloud/

[14] E.P. Shettle, "Models of aerosols, clouds and precipitation for atmospheric propagation studies", in Atmospheric propagation in the $U V$, visible, IR and mm-region and related system aspects AGARD Conference Proceedings No. 454, (1989) 
[15] M. Nicolet, "On the molecular scattering in the terrestrial atmosphere: an empirical formula for its calculation in the homosphere", Planet. Space Sci., Vol 32, pp 1467-1468 (1984)

[16] G.P. Anderson, et al, "AFGL Atmospheric Constituent Profiles $(0-120 \mathrm{~km}) "$ AFGLTR-86-0110, Hanscom AFB, MA 01736 (1986)

[17] L.C. Andrews, R.L. Phillips, "Laser Beam Propagation through Random Media", SPIE Press, Bellingham (WA), USA, 1998

[18] L.C. Andrews, R.L. Phillips, C.Y. Hopen, "Laser Beam Scintillation with Applications", SPIE Press, Bellingham (WA), USA, 2001

[19] J.A. Fleck, et al, "Time dependent propagation of High Energy Laser Beams through the Atmosphere", Applied Physics. Vol. 10, pp. 129-160 (1976)

[20] R. Jüngling, "Simulation gerichteter Ausbreitung optischer Wellen in turbulenter Atmosphäre", Diploma work, Institute for Communications and Navigation, DLR-Oberpfaffenhofen, May 2001 